\title{
Analysis and application of NAT in Dongguang blood center more than 10 years
}

\author{
Ziyi He, Shaobin Chen, LinYu, Qing Wang, Qingkai Chen, Jiaoli Zou, Jialin Che \\ Department of Clinical Laboratory, Dongguang Blood Center, Dongguan 523006, Guangdong, Chin
}

\begin{abstract}
This report reviewed the efficacy of nucleic acid testing (NAT), derived from assaying and measuring data, using a Cobass201 system at the Dongguan blood center from 2008 to 2017. During this period, four blood screening models,each reflecting procedure improvements designed to improve residual risk (RR) assessment were assessed. A total of 716846 blood donors were screened, detecting 1395 positive by the mixed pool test, which were finally modified to 900 positive cases, after final detection by the separation-single test: detecting 6 HIV cases, $4 \mathrm{HCV}$ cases and $890 \mathrm{HBV}$ cases with a total positive rate of $1.25 \%$. The lowest result was obtained from the twice administered enzyme-linked immunoassay (ELISA) test, used in conjunction with the single Cobas MPX v2.0 model, with rates of: 1/7 405 for HBV, 1/346 020 for HCV and 1/473 934 for HIV, respectively. NAT positive rate is not affected by different screening models. NAT is a recent detection method which can be used to good effect with ELISA, and is a worth while procedure for promoting blood transfusion safety.
\end{abstract}

Keywords: NAT, DNA, RNA, RR, HBV, HCV, HIV

\section{INTRODUCTION}

The major risk for blood transfusions is the potential spread of viral infection, notably HIV-1, HIV-2, $\mathrm{HCV}$ and HBV. As a new blood screening technology, namely nucleic acid testing (NAT) has been applied in many developed countries and regions, such as Japan, the United States, Germany, and so on. Due to the "window period" involved inenzyme-linked immunoassays (ELISA), the detection miss rate of HBV, HCV and HIV is one in ten thousand and one in amillion ${ }^{[1-6]}$. NAT, however can significantly shorten this virus detection window period (for HBV, HCV and HIV)by9, 59 and 11 days, respectively. At the same time, NAT can detect chronic or occult carriers with negative viral antibodies and detect

*Correspondence to: Ziyi He, MD, Department of Clinical Laboratory, Dongguang Blood Center, 19 Ningjing Road, Humen Town, Dongguan, Guangdong 523006, China.TEL: 86-769-85152673, E-mail: zyhe_8@163.com Conflict of interest:The authors declared no conflict of interests. antigen, as well as variant virus infection ${ }^{[7-13]}$. Unfortunately, there are still as mall number of donors who,despite being found risk negative by ELISA,still manage to infect recipients with their donated blood, with a limited amount of cases of hepatitis and AIDS being reported each year ${ }^{[14]}$. For example, in the United States,transfusion transmission risks for contracting HBV, HCV, and HIV are 1:66 000, 1:103 000, 1:676 000, respectively ${ }^{[15]}$. In China, NAT was introduced in 2004 and is still limited to a few blood centers, with few related reports as a consequence. In view of the very high NAT positive yield (about 1:700 1:2 000 in our blood center), there is a strong case for NAT to be universally applied in blood centers. However, the high HBV occult infection suggests that both a stricter donor selection and immunization may be good ways forward. From 2008, we began to study the application of the Cobas s201 NAT blood screening system, used four blood screening models and accumulated much experience contributing to this report. 


\section{MATERIALS AND METHODS}

\section{Sample source}

A total of 716846 blood donors's samples were collected and analyzed (after being stored at $2-8^{\circ} \mathrm{C}$ before screening) at the Dongguan blood center from 2008 to Dec $31^{\text {st }}, 2017$. This study was approved by the Dongguan Blood Center Ethics Committee and all aspects of the study complied with the Declaration of Helsinki.

\section{Reagents and instruments}

Cobas Ampliscreen, Cobas MPX test kit and Cobas MPX v2.0 reagents, as well as the Cobas s201 NAT screening system; were supplied by Roche Molecular Systems Inc.,CA.,USA. All ELISA reagents were produced by domestic or imported products.

\section{Methods and screening models}

We applied the Cobas NAT system to blood samples using the fluorescent labeling polymerase chain reaction (PCR) technique. The principle; under the catalysis of DNA polymerase, using 4 dNTPs to synthesize an exact copy of the template DNA, which is subjected to real-time fluorescence quantitative PCR reaction. As the PCR reaction proceeds, the PCR reaction's product accumulates and the intensity of the fluorescence signal also increases. This fluorescence intensity can be used to detect PCR production, and to obtain a fluorescence amplification curve. When the fluorescence signal turns from background to exponential growth phase and a positive fluorescence threshold is reached, the reverse is negative. To make sure blood products are safer, our blood center's laboratory applied several Cobas NAT systems and implemented four screening models, interfaced with various blood information management systems (Table 1).

Table 1 The development of NAT in ourblood center

\begin{tabular}{|c|c|c|c|}
\hline Period & Equipment & Screening models & Information system \\
\hline $2004-2008$ & Cobas Ampliscreen & $\begin{array}{l}\text { ELISA negative samples were collected and } 24 \text { samples were } \\
\text { manually mixed in one pool with the total of } 1000 \text { microlit- } \\
\text { ers.Manual preparation; reagent, and purified DNA/RNA, } \\
\text { centrifugation and sorting. Only amplification was by auto- } \\
\text { matic readingequipment. }\end{array}$ & $\begin{array}{l}\text { Ampliscreen included software, no infor- } \\
\text { mation system connecting. }\end{array}$ \\
\hline $2008-2009.9$ & Cobas s201 MPX v1.0 & $\begin{array}{l}\text { ELISA negative samples were collected;each group of } 6 \\
\text { samples were automatically mixed in one pool. Automatic } \\
\text { extraction, amplification and interpretation. Positive samples } \\
\text { in the mixed pool were separatedfor the single test. }\end{array}$ & $\begin{array}{l}\text { The results did not connected to the } \\
\text { Tangshan } 5.0 \text { information management } \\
\text { system. }\end{array}$ \\
\hline $2009.10-2013.7$ & Cobas s201 MPX v1.0 & $\begin{array}{l}\text { ELISA negative samples were mixed with } 6 \text { in } 1 \text { pool. Au- } \\
\text { tomatic extraction, amplification and interpretation .Positive } \\
\text { samples in the mixed pool were required to be separated for a } \\
\text { single test; to be completed within } 72 \text { hours. }\end{array}$ & $\begin{array}{l}\text { The results were connected to the Tang- } \\
\text { shan } 5.0 \text { information management system } \\
\text { and participated in the interpretation of } \\
\text { screening results. }\end{array}$ \\
\hline $2014.5-2014.9$ & Cobas s201 MPX v1.0 & $\begin{array}{l}\text { In order to improve the effective reagent utilization rate; } 108 \\
\text { samples per batch were carried out. }\end{array}$ & $\begin{array}{l}\text { The results were connected to the Qiao } 9.0 \\
\text { information management system. }\end{array}$ \\
\hline 2014.10-now & Cobas s201 MPX v2.0 & $\begin{array}{l}\text { The identification results werereported by MPX v } 2.0 \text { reagent. } \\
\text { Afull batch detection model of } 120 \text { samples was carried out } \\
\text { to improve the effective reagent utilization rate. }\end{array}$ & $\begin{array}{l}\text { The results were connected to the Qiao } 9.0 \\
\text { information management system. }\end{array}$ \\
\hline
\end{tabular}

Our early blood screening models were: HBsAg, anti-HCV, anti-HIV, and anti-TP, which were detected using two different ELISA reagents from different manufacturers. Later, the third generation of ELISA reagents further improved the quality of detection. With the fourth generation of ELISA reagents, improvements such as P24 antigen made HIV infection detectable. The NAT method was also improved; from the manual mixing of 24 samples, and the manual extraction of viral nucleic acids, to the use of automated equipment for mixing 6 samples with the automatic extraction of viral nucleic acids. The latest NAT equipment achieves full automation; the information system is more perfect, and data transmission is faster. Cobas MPX reagents have also been updated several times, from the earliest manual kits to automated kits (Cobas MPX v1.0 v2.0), with the second generation having higher sensitivity and specificity. During the process of improvement our blood center has used a total of four screening models. The screening models in this article refer to testing and method simplemented only in the laboratory, and do not include prelimi- 
nary screening prior to blood collection. The samples in our laboratory were firstly tested with two different ELISA reagents, and their $\mathrm{ABO} / \mathrm{RhD}$ group was identified. The reactive samples in the first ELISA tests underwent retests on double samples on original tube and blood-sampling bag, if it was again found reactive, NAT was not performed. All samples with nonreactive ELISA results after undergoing NAT testing (on Cobas s201 testing equipment)were mixed into one pool, at 6 samples per group. If the mixed pool was reactive, all mixed samples were separated for another single test; if the single test was reactive, the sample was determined to be reactive, and if the single test was non-reactive, the sample was determined to be non-reactive; if the pool was non-reactive, the final results of all 6 mixed samples were all deemed negative; if a sample was positive whenthe Cobas MPX vl.0 reagent was used, further identification of the virus type was required, however when the Cobas MPX v2.0 was used, this was not necessary.

\section{Risk assessment}

Residual risk (RR) refers to the possibility of a recipient being infected by a transfusion-transmitted virus from the transfusion of a "qualified" blood component (after transfusion-transmitted viral screening). The use of NAT in blood donor screening has been implemented in many countries to reduce the RR. NAT can shorten the"window period", thereby offering blood centers a much higher sensitivity for detecting viral infections. The estimation of RR is essential to monitor and improve blood safety. Risk assessment is based on mathematical models.

\section{Statistical analysis}

SPSS13.0 was used for statistical analysis, and the $\chi^{2}$ test was used to determine the positive frequency of four screening models in different phases.The significance level is $P<0.05$.

\section{RESULTS}

\section{Basic situation}

A total of 716846 donors' samples were screened by NAT over 10 years, 1395 positive results were de- tected by the mixed pool test which was corrected to 900 positive cases after re-examination by the separation-single test $(61 \%)$, including $6 \mathrm{HIV}$ cases, $4 \mathrm{HCV}$ cases and $890 \mathrm{HBV}$ cases; the total positive rate was $1.25 \%$ (Table 2 and Table 3).

Table 2 The scrap rate for unqualified blood by NAT from 2008 to 2017

\begin{tabular}{lrcccc}
\hline Year & $n$ & $\begin{array}{c}\text { Pooling } \\
\text { positive }\end{array}$ & $\begin{array}{c}\text { Split } \\
\text { positive }\end{array}$ & $\begin{array}{c}\text { Split positive } \\
\text { rate }(\%)\end{array}$ & $\begin{array}{c}\text { Scrap } \\
\text { rate }(\%)\end{array}$ \\
\hline 2008 & 54015 & 56 & $29^{*}$ & 0.52 & 0.54 \\
2009 & 60369 & 116 & $61^{*}$ & 0.53 & 1.01 \\
2010 & 68170 & 128 & 91 & 0.71 & 1.33 \\
2011 & 71910 & 122 & 89 & 0.73 & 1.24 \\
2012 & 75865 & 118 & 88 & 0.75 & 1.16 \\
2013 & 78563 & 179 & 117 & 0.65 & 1.49 \\
2014 & 775781 & 139 & 91 & 0.65 & 1.20 \\
2015 & 69459 & 159 & 100 & 0.63 & 1.27 \\
2016 & 78796 & 180 & 110 & 0.61 & 1.39 \\
2017 & 83918 & 198 & 124 & 0.63 & 1.47 \\
Total & 716846 & 1395 & 900 & 0.61 & 1.25 \\
\hline
\end{tabular}

*we completed NAT on some blood donors sample from Jan 1, 2008 to Oct 12, 2009,NAT results were not included in the system's interpretation of blood safety. Therefore, the NAT data for this period are not included in the statistics in table 3

From 2012 to 2017, the total scrap rate and NAT positive rate is seen in Fig.1. The total positive rate decreased, due to improved blood donation screening models. However, results still showed that blood sources' safety could be increased. To achieve this, the laboratory strengthened the management and standardization of ALT detection. The total positive rate was also decreased by using POCT (point-of-care Testing) in the collection department. NAT positive rates were stable from 2012 to 2017 , as the ELISA models didn't affect them (Fig.1). Meanwhile, owing to enclosed detection, and the stability of NAT system, as table positive rate of detection was ensured.

\section{$\mathbf{R R}$ assessment}

The assessment results showed that the screening model with the highest RR results were obtained from the twice applied ELISA method; The HBV RR range was from 1/5 429 to $1 / 8$ 281, while the HCV RR range was form 1/15 599 to $1 / 346020$. As a confirmatory test wasn't performed on the $\mathrm{HCV}$ positive reaction

Table 3 Fourscreening modelsin different phases

\begin{tabular}{|c|c|c|c|c|c|c|}
\hline \multirow{2}{*}{ Phases } & \multirow{2}{*}{ Abbreviation } & \multirow{2}{*}{$n$} & \multirow{2}{*}{$\begin{array}{c}\text { NAT Positive } \\
{[n(\%)]}\end{array}$} & \multicolumn{3}{|c|}{ Identification } \\
\hline & & & & $\mathrm{HBV}$ & $\mathrm{HCV}$ & HIV \\
\hline & & & & I & I & I \\
\hline 2013.08.02 2016.04.08 & 1 ELISA+NAT(v1.0) & 201342 & $262(0.130)$ & 257 & 1 & 4 \\
\hline $2016.04 .09 \sim 2017.12 .31$ & 2 ELISA+NAT(v2.0) & 145478 & $216(0.148)$ & 214 & 2 & 0 \\
\hline
\end{tabular}

$x^{2}=7.43, P=0.05$. 
samples; this may be a false positive. On the screening models, the twice administered ELISA, plus singly applied NAT (Cobas MPX v2.0) had the lowest RR for $\mathrm{HCV}$, which was related to sensitivity and specificity improved by 1) thereplacement of ELISA reagents from the $3^{\text {rd }}$ generation to the $4^{\text {th }}$ generation and 2) NAT reagentupgrades from the $1^{\text {st }}$ generation to the $2^{\text {nd }}$ generation. The RR of the twice applied ELISA was the highest, and the RR was reduced to $1 / 364020$ by using ELISA twice plus NAT once(Cobas MPX v2.0). The RR of HIV was from $1 / 400000$ to $1 / 546448$, and wasn't affected by the screening models (Table 4).

\section{DISCUSSION}

In 1997, Netherlands and Germany began to apply NAT in blood screening, However Japan was the first country in the world to apply NAT for routine blood screening. In 1999, Singapore, the United States, and other countries also began, with France beginningin $2000^{[5]}$. In China, the NAT test for transfusion-transmitted viruses was successively applied in Xiamen, Shandong, Shanghai, Shenzhen with others joining in early 2000 , there by accumulating much experience in the construction of NAT laboratories, quality management, personnel training and screening models, while significantly reducing the risk of transfusion-transmitted viruses. NAT is able to detect pathogenic nucleic acids, has high sensitivity, specificity, and throughput and is able to detect viruses missed by other tests, during the "window period" concerning virus mutation and a typical seroconversion (such as occult infections). Because of this, NAT blood screening has been defined as an essential requirement in China, as

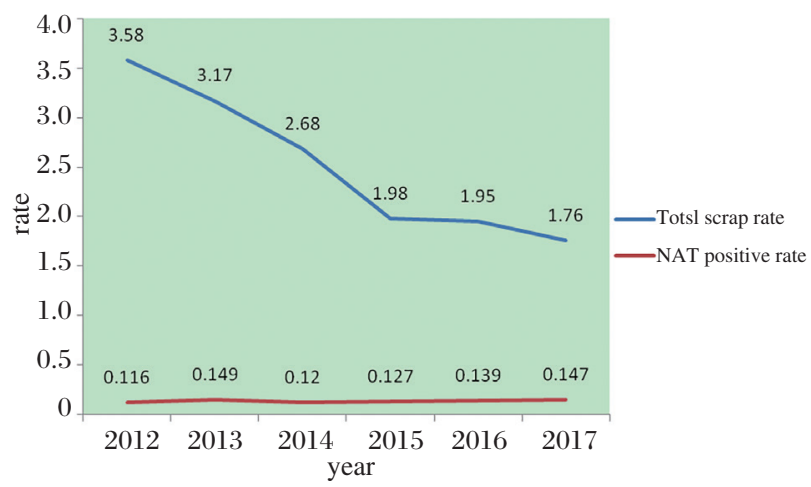

Fig.1 Trends of the total scrap rate and positive rate of NAT in blood screening from 2012 to 2017

Table 4 Estimation of RR after 4 screening models in Dongguan (1 U of Blood per Transfusion)

\begin{tabular}{|c|c|c|c|c|c|c|}
\hline \multirow{2}{*}{ Screening models } & \multicolumn{2}{|c|}{$\mathrm{HBV}$} & \multicolumn{2}{|c|}{$\mathrm{HCV}$} & \multicolumn{2}{|c|}{ HIV } \\
\hline & $P$ value & $\mathrm{RR}$ & $P$ value & RR & $P$ value & RR \\
\hline 2 ELISA & $184.18 \times 10^{-6}$ & $1 / 5429$ & $64.11 \times 10^{-6}$ & $1 / 15599$ & $1.83 \times 10^{-6}$ & $1 / 546448$ \\
\hline 1ELISA+1 NAT(v1.0) & $120.77 \times 10^{-6}$ & $1 / 8281$ & $4.41 \times 10^{-6}$ & $1 / 226757$ & $2.36 \times 10^{-6}$ & $1 / 423723$ \\
\hline 2ELISA+1 NAT(v2.0) & $135.03 \times 10^{-6}$ & $1 / 7405$ & $2.89 \times 10^{-6}$ & $1 / 346020$ & $2.11 \times 10^{-6}$ & $1 / 473934$ \\
\hline
\end{tabular}

shown by the Chinese National Health Commission's requirement touse NAT after 2016.

China as a region, has the highest prevalence of HBV, so the Dongguan blood center was privileged to become the first to apply the Cobas NAT system, and the first blood center to reference its results in China, using Roche diagnostic equipment, providing training and demonstrations to many Chinese blood specialists.

With the development of a variety of blood safety screening models, laboratories are now able to utilize the advantages of ELISA in combination with NAT. After applying NAT for nearly 10 years, our ongoing analysis found that the positive rate of transfusiontransmitted HBV/HCV/HIV by twice applied ELISA plus singly administered NAT was higher than that of singly applied ELISA (plus once applied NAT) ${ }^{[16]}$. However, the positive rate by ELISA and NAT both applied once, was higher than that of the two times of ELISA plus one time of NAT in the early pe$\operatorname{riod}^{[17]}$. This showed the importance of the reagent, as later reagents improved. The application of NAT can shorten the window period and reduce the RR of transfusion transmitted virus from $40 \%$ to $50 \%^{[18,}$ ${ }^{19]}$. Experience also showed that blood safety screening models of the twice applied ELISA, plus once administered NAT (Cobas MPX v2.0) can effectively reduce the RR. Screening models that employed NAT after one or two times ELISA, had positive rate which reached $0.14 \%$, with 4 cases of $\mathrm{HCV}$ and 6 cases of HIV detected. This was extremely important for safety,but also shows that the rate of missed detection with ELISA is very high, which needs to be made up by NAT. However NAT also has detection error rate: its false positive rateis 1:22 000 donors, which exceeds its true positives (HIV: 1:4.13 million; HCV: 1:0.31 million). The cause of these false positives has been confirmed to be laboratory contamination, rather than from within the samples themselves ${ }^{[20]}$. Studies have shown that some samples had a NAT positive result for their first test, but returned negative under re-examination. Interestingly however, they were still positive with HBV-DNA after ultracentrifugation, 
owing to the extremely low concentration of virus in the samples.

So, is it safe to rely on NAT? NAT cannot completely replace ELISA. Several studies have shown that serologically positive samples did not test $100 \%$ positive using NAT. This is because NAT only shortens the "window period" for HBV, HCV, and HIV infection. So using NAT samples in the "window period", viruses can still be missed,causing RR, irrespective of the screening method used. Further literature in China reported RR assessment using the mathematical model for HIV, in which; Shijiazhuang City was $1 / 251889^{[21]}$ and Qingdao City $1 / 186512^{[22]}$. Both of which were higher than $1 / 40000$ to $1 / 546448$ in the present study, however this study was basically consistent with the $1 / 45872^{[23]}$ reported by Shenzhen City. This may be related to the prevalence of the virus among regions. Seed et $\mathrm{al}^{[24]}$ reported RR from the Australian Red Cross Blood Center as: HIV 1/3 415 000, HCV 1/911 000, and HBV 1/483 000. Our finding was also higher than the RR rates that Seed et $\mathrm{al}^{[25]}$ reported three years later: 1/339 000 for HBV, 1/3 636000 for HCV and 1/7 299000 for HIV. Our RR figures were higher than those found in foreign countries; especially HIV, which was markedly higher.

Summarizing our experience with the four screening models; results showed that models using the twice applied ELISA test attained the highest RR. Twice applied ELISA plus singly applied NAT (Cobas MPX v2.0) had the lowest RR for HCV. The RR of twice applied ELISA was the highest, with the RR being reduced to $1 / 364020$ by using ELISA twice plus NAT once (Cobas MPX v2.0). The RR of HIV was from 1/400 000 to $1 / 546448$, and wasn't affected by screening models. The RR assessment of all mentioned transfusion-transmitted viruses were higher than those found in foreign countries, especially the HIV RR, which was much higher than reported in other domestic reports. The main reasons for these we believe are as follows: firstly, migrant workers in Dongguan are the main source of blood donors, who lack a basic understanding of sexually transmitted diseases and their prevention. Secondly,the sensitivity and specificity of detection reagents have been continuously improved, leading to an increased positive rate. Thirdly,some donors, especially some infected with HIV, present at donation facilities precisely for the purpose of HIV detection, thus greatly increasing the HIV positive rate.

At present, China has the conditions to comprehensively perform NAT, having all the necessary technology, personnel qualifications, and quality manage- ment. The main constraint is the implementation of testing funds, and financial support, which in many local areas is insufficient. The cost of NAT is expensive, and it has been proposed that screening models with once applied ELISA plus one cycle of NAT, should be adopted to solve the problem. However, higher requirements are needed for the sensitivity of reagents, for which labs are awaiting further verification. Post transfusion RR is objective, but it can be controlled and reduced, establishing a screening model suitable for national conditions, that can not only reduce the economic burden, but also minimize the RR. To do this, reagents need to be carefully evaluated as having high sensitivity and specificity, used together with reliable equipment. At the same time, it is necessary to strengthen publicity and education, with the recruitment and selection oflow-risk donors.

\section{Acknowledgements and funding}

Ziyi He and all authors performed the research and contributed to the clinical application. This work was supported by the Dongguan Social Development Science and Technology Key Project (201750715026436). We certify that we have no affiliation with or financial involvement in any organization or entity with a direct financial interest in the subject matter or materials discussed in this manuscript. The manuscript has been seen and approved by all authors, it is not under active consideration for publication, has not been accepted for publication, nor has it been published.

\section{References}

[1] Mine H, Emura H, Miyamoto M, et al. High throughput screening of 16 million serologically negative blood donors for hepatitis $\mathrm{B}$ virus, hepatitis $\mathrm{C}$ virus and human immunodeficiency virus type-l by nucleic acid testing with specific and sensitive multiplex reagent in Japan[J]. $J$ Virol Methods, 2003, 112(1/2):145-151

[2] Hourfar MK, Jork C, Schottsted V, et al. Experience of germ an Red Cross blood donor services with nucleic acid testing:results of screening more than 30 million blood donations for human immunodeficiency virus1,hepatitis $\mathrm{C}$ virus, and hepatitis B virus[J].Transfusion, 2008, 48(8):1558-1566

[3] Nübling C, Heiden M, Chudy M, et al. Experience of mandatory nucleic acid test(NAT)screening across all blood organizations in Germany:NAT yield versus breakthrough transmissions[J].Transfusion, 2009, 49(9):1850-1858

[4] Stramer SL, Wend U, Candotti D, et al. Nucleic acid testing to detect HBV infection in blood donors[J].N Engl J Med,2011,364(3):236-247

[5] Taira R, Satake M, Momose S, et al.Residual risk of transfusion-transmitted hepatitis B virus (HBV) infection 
caused by blood components derived from donors with occult HBV infection in Japan[J]. Transfusion, 2013, 53(7):1393-1404

[6] Saber HR, Tabatabaee SM, Abasian AA, et al. Incidence and residual risk of $\mathrm{HIV}, \mathrm{HBV}$ and $\mathrm{HCV}$ infections among blood donors in Tehran[J]. Indian J Hematol Blood Transfus, 2017, 33(3):412-416

[7] Renfurong. Application of NAT in blood screening at domestic and abroad[J]. Beijing Medical Journal, (in Chinese)2008, 30(8):561-564.

[8] Rocha D, Andrade E, Godoy DT, et al. The Brazilian experience of nucleic acid testing to detect human immunodeficiency virus, hepatitis $\mathrm{C}$ virus, and hepatitis B virus infections in blood donors[J]. Transfusion, 2018,58(4):862-870

[9] Men SS, Lv LZ, Yf C, et al. Blood test patterns for blood donors after nucleic acid detection in the blood center[J]. Zhongguo Shi Yan Xue Ye Xue Za Zhi, (in Chinese)2017, 25(6):1815-1819.

[10] Galel SA, Simon TL, Williamson PC. et al, sensitivity and specificity of a new automated system for the detection of hepatitis B virus,hepatitis C virus, and human immunodeficiency virus nucleic acid in blood and plasma donations[J]. Transfusion, 2018, 58(3):649-659

[11] Ghosh K, Mishra K. Nucleic acid amplification testing in Indian blood banks:A review with perspectives[J]. Indian J Pathol Microbiol, 2017, 60(3):313-318

[12] Lin H, Chen X, Zhu SW, et al. Prevalence of occult hepatitis $\mathrm{C}$ virus infection among blood donors in Jiangsu, China[J]. Intervirology, 2016, 59(4):204-210

[13] Duskova D, Darebnicek L. Nucleic acid testing of hepatitis B virus, hepatitis $\mathrm{C}$ virus, and human immunodeficiency virus 1, 2 in blood donors in the General University Hospital, Prague[J]. Acta Virol, 2014, 58(2):146-151

[14] Candotti D, Boizeau L, Laperche S. Occult hepatitis B infection and transfusion-transmission risk[J]. Transfusion Clinique et Biologique, 2017, 24(3):189-195

[15] Bai Y, ChangWeihong.The course and actualities of nudeic acid testing for blood screening in the United States[J]. Int J Biologicals, 2009, 32(2):101-105
[16] Fei YT, HE ZY, WANG DW, et al. Comparative study on two detection scheme of voluntary blood donors infected HBV/HCV/HIV[J].Journal of Modern Laboratory Medicine,2014,29(3):13-138

[17] HE ZY, YU L, Wang Q, et al. Analysis of nucleic acid amplification technology in different screening modes[J]. Chin J Blood Transfusion, 2016, 29(7):693-695

[18] Alvarez M, Oyonarte S, Rodríguez PM, et al. Estimated risk of transfusion-transmitted viral infections in Spain[J]. Transfusion, 2002, 42(8):994-998

[19] Kumar R, Gupta S, Kaur A, et al.Individual donornucleic acid testing for human immunodeficiency virus1,hepatitis $\mathrm{C}$ virus and hepatitis B virus and its role in blood safety[J]. Asian J Transfus Sci,2015,9(2):199-202

[20] Gou H, Pan Y, Ge H, et al. Evaluation of an individualdonation nucleic acid amplification testing algorithm for detecting hepatitis B virus infection in Chinese blood donors[J]. Transfusion, 2015, 55(9):2272-2281

[21] Zhang J, Wangsuling, Zhaolihua, et al. HIV infection rate and residual risk assessment after blood screening of voluntary blood donors in Shijiazhuang area from 2010 to 2015[J].J Clin Hematol,2016,10(29):812-813

[22] Feng Q, Yang ZS, Pan HP, et al. Evaluation of the residual risk of HIV prevalence and blood screening for the first/repeated donors in Qingdao[J]. Chin J Blood Transfusion, 2012, 25(9):868-869

[23] Wang SX, Zeng JF, Yang AL, et al.Investigation and analysis of HIV infection and residual risk assessment of blood transfusion in shenzhen[J].Chin J Blood Transfusion, 2012, 25(7):674-676

[24] Seed CR, Cheng A, Ismay SL, et al.Assessing the accuracy of three viral risk models in predicting the outcome of implementing HIV and HCVNAT donor screening in Australia and the implications for future HBVNAT[J]. Transfusion,2002,42(10):1365-1372

[25] Seed CR, Kiely P, Keller AJ. Residual risk of transfusion transmitted human immunodeficiency virus, hepatitis $\mathrm{B}$ virus,hepatitis $\mathrm{C}$ virus and human $\mathrm{T}$ lymphotrophic virus[J]. Intern Med J, 2005, 35(10):592-598.

(Received 08 April 2018, Revised 17 May 2018, Accepted 30 May 2018) 